\title{
Measurement of the $\gamma^{*} \gamma^{*} \rightarrow \eta^{\prime}$ transition form factor and of the $e^{+} e^{-} \rightarrow \pi^{+} \pi^{-} \pi^{0} \pi^{0} \pi^{0}$ and $\pi^{+} \pi^{-} \pi^{0} \pi^{0} \eta$ cross sections by BABAR
}

\author{
J. William Gary ${ }^{1, *}$ \\ ${ }^{1}$ Department of Physics and Astronomy, University of California, Riverside, CA 92521, USA
}

\begin{abstract}
Two recent studies from the BABAR experiment at SLAC on lowenergy hadronic final states in $e^{+} e^{-}$annihilations are presented. The first study provides the first-ever measurement of the $\gamma^{*} \gamma^{*} \rightarrow \eta^{\prime}$ transition factor, where $\gamma^{*}$ denotes an off-shell photon. The second study provides the first-ever measurements of the $e^{+} e^{-} \rightarrow \pi^{+} \pi^{-} \pi^{0} \pi^{0} \pi^{0}$ and $\pi^{+} \pi^{-} \pi^{0} \pi^{0} \eta$ cross sections, including studies of the intermediate resonance states and the corresponding $J / \psi$ and $\psi(2 S)$ branching fractions.
\end{abstract}

\section{Introduction}

The BABAR experiment at SLAC operated at the PEP-II asymmetric-energy electron positron collider between 1999 and 2008. Despite having ceased operation 10 years ago, the data analysis is still very active. The BABAR data set is still rather unique and continues to provide important new information. There have been seven BABAR papers submitted to journals so far in 2018, with three or four more submissions expected before the end of the year. The BABAR detector provided precise measurements of momentum for charged tracks and had excellent particle identification capabilities. Although it was primarily designed for the study of $\mathrm{CP}$ violation in $B$ meson decays, its general purpose design made it suitable for a wide variety of other physics studies, such as those discussed here. The vast majority of the BABAR data was collected at the peak of the $\Upsilon(4 \mathrm{~S})$ resonance. The analyses presented here make use of $470 \mathrm{fb}^{-1}$ of data collected at or $40 \mathrm{MeV}$ below the $\Upsilon(4 \mathrm{~S})$ peak, corresponding to an $e^{+} e^{-}$center-of-mass (c.m.) energy of about $10.6 \mathrm{GeV}$.

\section{The $\gamma^{*} \gamma^{*} \rightarrow \eta^{\prime}$ transition form factor}

The first topic presented here is a measurement of the $\gamma^{*} \gamma^{*} \rightarrow \eta^{\prime}$ transition form factor (TFF). This result was recently submitted to Physical Review D [1]. A Feynman diagram is shown in Fig. 1. This is a two photon process, in which both the initial electron and initial positron emit a highly virtual photon. The two virtual photons combine to produce the $\eta^{\prime}$ meson. The electron and positron are both scattered away from the beam direction and are observed in the detector. This, the two-photon events used in this analysis are double tagged.

The TFF is defined via the amplitude $T$ for the $\gamma^{*} \gamma^{*} \rightarrow \eta^{\prime}$ transition:

$$
T=-i 4 \pi \alpha \epsilon_{\mu \nu \beta \gamma} \epsilon_{1}^{\mu} \epsilon_{2}^{\nu} q_{1}^{\beta} q_{2}^{\gamma} F_{\eta^{\prime}}\left(Q_{1}^{2}, Q_{2}^{2}\right)
$$

\footnotetext{
*e-mail: bill.gary@ucr.edu
} 


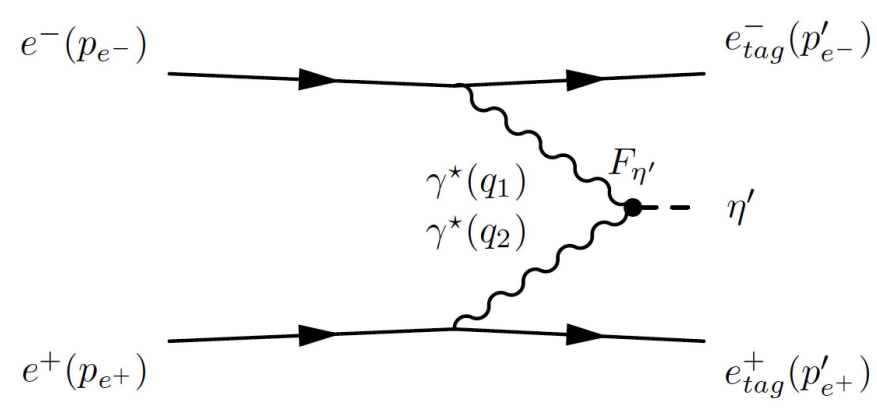

Figure 1. Feynman diagram for the two-photon process $e^{+} e^{-} \rightarrow e^{+} e^{-} \eta^{\prime}$.

where $\alpha$ is the fine structure constant, $\epsilon_{1,2}$ and $q_{1,2}$ are the polarization vectors and 4-momenta, respectively, of the two virtual photon, $\epsilon_{\mu \nu \beta \gamma}$ is the Levi-Civita tensor, and $F_{\eta^{\prime}}$ is the TFF. The photons are space-like and so the $q_{1,2}^{2}$ are negative. It is thus convenient to define $Q_{1,2}^{2}=-q_{1,2}^{2}$ so that the arguments of the TFF are positive.
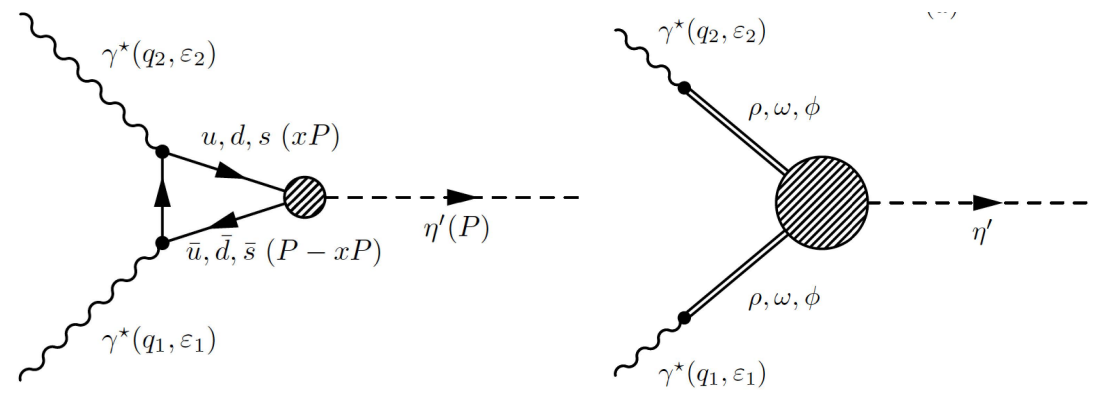

Figure 2. Diagrams illustrating the (left) perturbative QCD and (right) vector meson dominance model descriptions of the $\gamma^{*} \gamma^{*} \rightarrow \eta^{\prime}$ process.

The TFF describes the effect of the strong interaction on the $\gamma^{*} \gamma^{*} \rightarrow \eta^{\prime}$ reaction, and the measurement of the $\gamma^{*} \gamma^{*} \rightarrow \eta^{\prime}$ TFF can be used to provide a novel test of QCD. Two models have been used to describe the TFF: perturbative QCD (pQCD) and the vector meson dominance (VMD) model. Diagrams illustrating the $\gamma^{*} \gamma^{*} \rightarrow \eta^{\prime}$ transition in the two models are shown in Fig. 2. The hatched circles in the two diagrams indicate the QCD interactions that are described by the TFF.

Two previous measurements of the TFF of the $\eta^{\prime}$ have been presented: one from the CLEO experiment [2] and a more recent and precise one from BABAR [3]. However, these two studies were based on the single-tag mode $\gamma^{*} \gamma \rightarrow \eta^{\prime}$, with only one off-shell photon. For only one off-shell photon, $\mathrm{pQCD}$ and the VMD model both predict the same asymptotic behavior for the TFF, namely $F_{\eta^{\prime}}\left(Q^{2}\right) \sim 1 / Q^{2}$, and therefore the two models cannot be distinguished. In contrast, for two off-shell photons, as in the present study, the pQCD and VMD asymptotic predictions differ and therefore the pQCD result can be tested: $F_{\eta^{\prime}}\left(Q_{1}^{2}, Q_{2}^{2}\right) \sim 1 /\left(Q_{1}^{2}+Q_{2}^{2}\right)$ for pQCD and $\sim 1 /\left(Q_{1}^{2} Q_{2}^{2}\right)$ for VMD.

The events are reconstructed through the $e^{+} e^{-} \rightarrow e^{+} e^{-} \eta^{\prime} \rightarrow e^{+} e^{-} \pi^{+} \pi^{-} \eta$ decay chain, with $\eta \rightarrow \gamma \gamma$. Thus, events with an identified $e^{+} e^{-}, \pi^{+} \pi^{-}$, and $\gamma \gamma$ pair are selected, making use of the BABAR particle identification capabilities. The $\gamma \gamma$ pair mass is required to be 
consistent with the $\eta$ mass. A kinematic fit of the $\gamma \gamma$ pair is then performed, with a constraint to the $\eta$ mass, to improve the photon momentum measurements. The $\pi^{+} \pi^{-} \eta$ invariant mass is then required to be consistent with the $\eta^{\prime}$ mass. The total c.m. momentum and energy of the $e^{+} e^{-} \eta^{\prime}$ candidate system is then required to be consistent with zero and the $e^{+} e^{-}$c.m. energy, respectively.
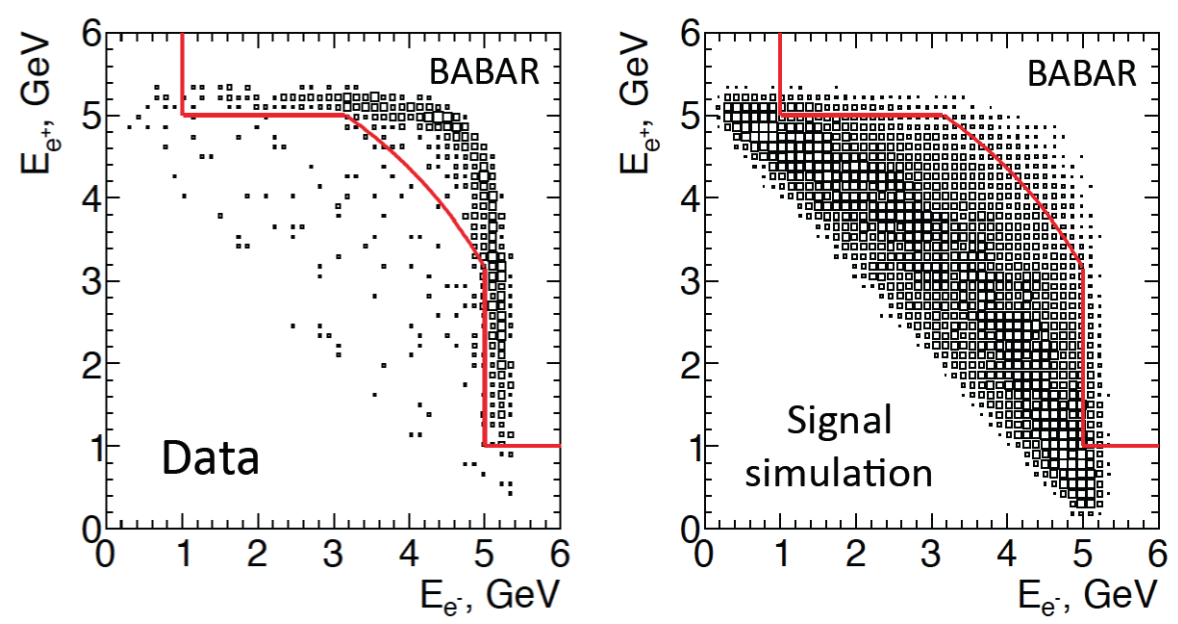

Figure 3. Scatter plots for (left) data and (right) signal event simulation of the final-state positron c.m. energy versus the final-state electron c.m. energy. Events below and to the left of the solid (red) line are retained.

Background is rejected with selection criteria applied in the plane of the final-state $e^{+}$and $e^{-}$c.m. energies $E_{e^{+}}$and $E_{e^{-}}$. The scatter plots for the data and for Monte Carlo (MC) signal simulation are shown in Fig. 3. The data, dominated by background at this stage, demonstrate that, for background events, either the final state $e^{+}$or $e^{-}$usually has the full beam energy of the initial $e^{+}$or $e^{-}$. The signal simulation shows that, for signal events, both the $e^{+}$and $e^{-}$ have usually lost much of their initial energy. Signal events are selected by retaining events below and to the left of the red solid line.

Figure 4 shows the distribution of the $\eta$ versus the $\eta^{\prime}$ candidate mass for the selected events. The distributions are shown both for the (left) data and (right) signal simulation. Within the signal box, shown by the rectangle at the center, there is a significant clustering of events in the data, consistent with the expectation from the signal simulation. Thus, a clear signal is seen. The background is small. Only events within the signal box are retained for further analysis.

The selected events are binned in five intervals of $Q_{e^{-}}^{2}$ versus $Q_{e^{+}}^{2}$ as illustrated in Fig. 5 (left). Because of the symmetry in the theoretical expressions under the exchange of the electron and positron, intervals 3 and 4 each contain two disjoint regions, mirror symmetric with respect to the diagonal. The number of signal events in each of the five regions is determined from a likelihood fit to the $\eta^{\prime}$ candidate mass distribution. The signal shape is taken from simulation. The background shape is taken to be linear. As examples, the fit results for regions 1 and 3 are shown in Fig. 5 (right). Summed over all intervals, 46.2 $2_{-7.0}^{+8.3}$ signal events are obtained, corresponding to a significance of around 6 standard deviations. 

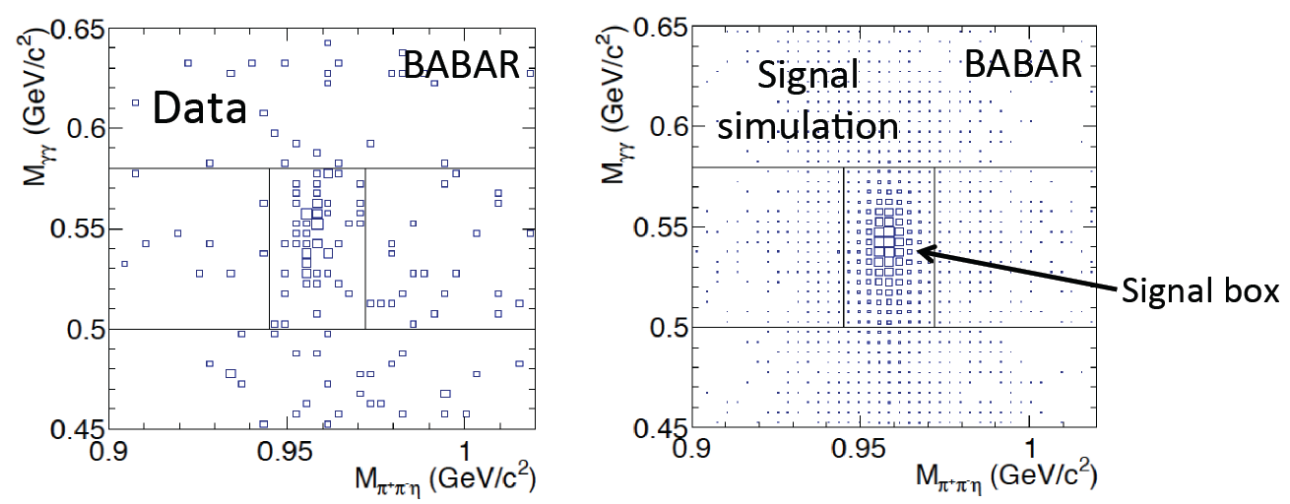

Figure 4. Scatter plots for (left) data and (right) signal event simulation of the candidate $\eta$ mass versus the candidate $\eta^{\prime}$ mass. The signal box is indicated in the figure.
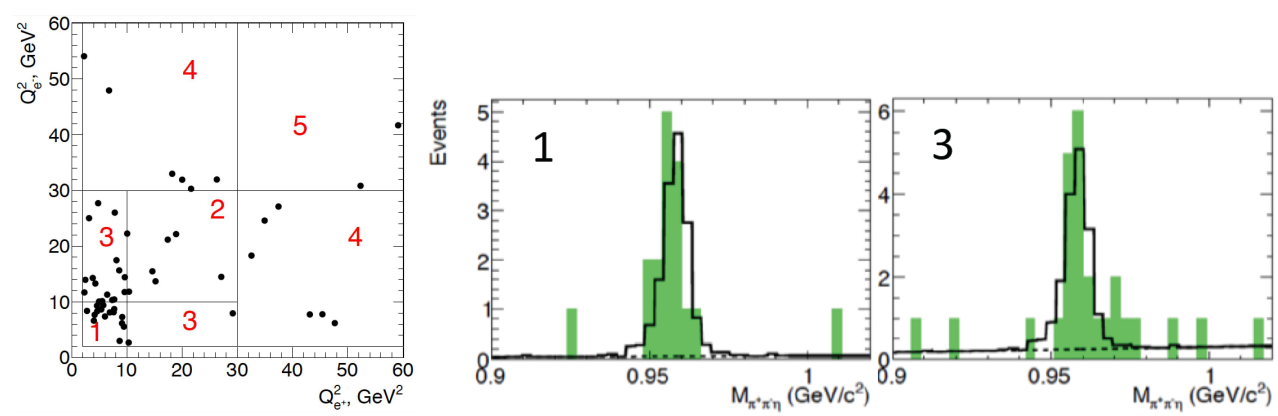

Figure 5. (left) The intervals in $Q_{e^{-}}^{2}$ versus $Q_{e^{+}}^{2}$ used for the $\gamma^{*} \gamma^{*} \rightarrow \eta^{\prime}$ TFF measurement. (right) Distribution of data (solid, green histograms) and fit results (solid curve) for the signal and background components in regions 1 and 3 . The dashed curve shows the fitted prediction for the background.

The double-differential cross section

$$
\frac{d^{2} \sigma}{d Q_{1}^{2} d Q_{2}^{2}}=\frac{1}{\epsilon R \mathcal{L} \mathcal{B}} \frac{d^{2} N}{d Q_{1}^{2} d Q_{2}^{2}}
$$

is measured in each of the five intervals, where $\epsilon$ is the detection efficiency, $R$ is a radiative correction accounting for initial-state photon radiation, $\mathcal{L}$ is the integrated luminosity, and $\mathcal{B}$ is the product of the $\eta^{\prime} \rightarrow \pi^{+} \pi^{-} \eta$ and $\eta \rightarrow \gamma \gamma$ branching fractions. The measured $\gamma^{*} \gamma^{*} \rightarrow \eta^{\prime}$ TFF, denoted $F^{2}\left(Q_{1}^{2} Q_{2}^{2}\right)$, is given by

$$
F^{2}\left(Q_{1}^{2} Q_{2}^{2}\right)=\frac{\left[d^{2} \sigma /\left(d Q_{1}^{2} d Q_{2}^{2}\right)\right]_{d a t a}}{\left[d^{2} \sigma /\left(d Q_{1}^{2} d Q_{2}^{2}\right)\right]_{M C}} F_{\eta^{\prime}}^{2}\left(Q_{1}^{2} Q_{2}^{2}\right)
$$

where $F_{\eta^{\prime}}^{2}\left(Q_{1}^{2} Q_{2}^{2}\right)$ is the predicted TFF from simulation. The nominal $F_{\eta^{\prime}}^{2}\left(Q_{1}^{2} Q_{2}^{2}\right)$ is based on pQCD. Consistent results for $F^{2}\left(Q_{1}^{2} Q_{2}^{2}\right)$ are obtained using the VMD prediction for $F_{\eta^{\prime}}^{2}\left(Q_{1}^{2} Q_{2}^{2}\right)$. The model uncertainty is evaluated by repeating the calculation assuming a constant TFF.

The results for the $\gamma^{*} \gamma^{*} \rightarrow \eta^{\prime}$ TFF in the five intervals of Fig. 5 (left) are shown in Fig. 6. The pQCD predictions at leading order (LO) and next-to-leading order (NLO) are shown by 


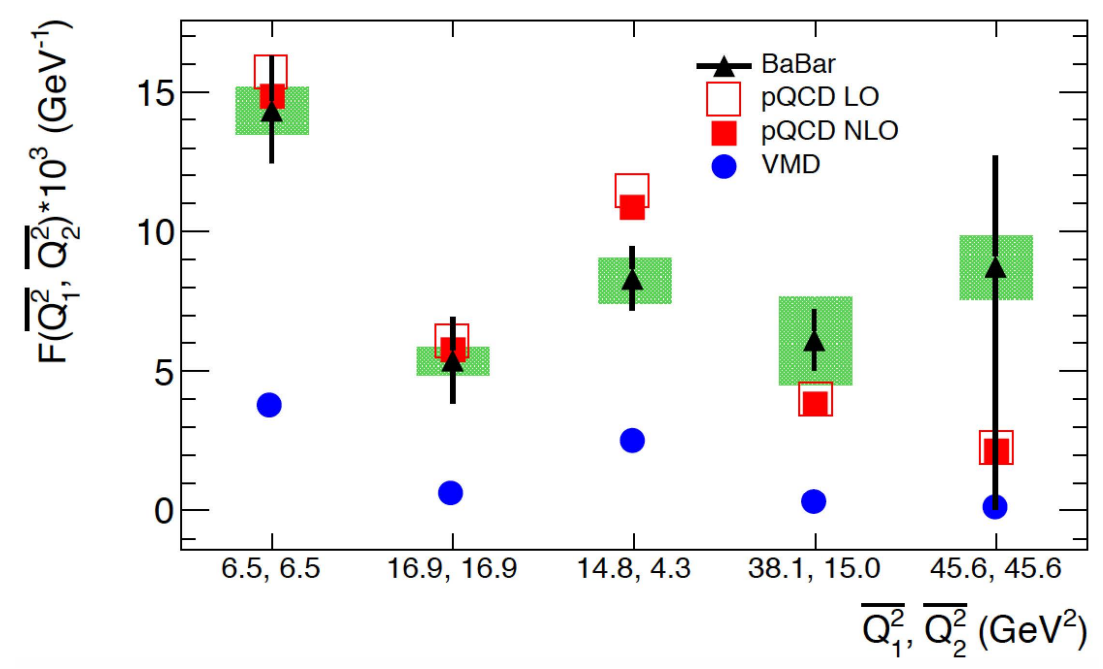

Figure 6. The measured $\gamma^{*} \gamma^{*} \rightarrow \eta^{\prime}$ transition form factor (triangles) in the five intervals of $\left(Q_{1}^{2}, Q_{2}^{2}\right)$ in comparison with the predictions of pQCD at leading- (open squares) and next-to-leading- (filled squares) order and with the predictions of the vector meson dominance model. The error bars on the data points are statistical. The (green) rectangular shaded areas show the systematic uncertainties.

the open and filled (red) squares, respectively. The data are seen to generally agree with the predictions of $\mathrm{pQCD}$. Higher order corrections have only a small impact on the $\mathrm{pQCD}$ predictions. The VMD predictions are shown by the filled (blue) circles and generally are in strong disagreement with the data.

\section{The $e^{+} e^{-} \rightarrow \pi^{+} \pi^{-} \pi^{0} \pi^{0} \pi^{0}$ and $\pi^{+} \pi^{-} \pi^{0} \pi^{0} \eta$ cross sections}

The second topic presented here is a measurement of the $e^{+} e^{-} \rightarrow \pi^{+} \pi^{-} \pi^{0} \pi^{0} \pi^{0}$ and $\pi^{+} \pi^{-} \pi^{0} \pi^{0} \eta$ cross sections. This result is still preliminary. It is to be submitted to Phys. Rev. D sometime soon. This study represents the latest chapter in the long-standing and extensive BABAR program to measure all possible exclusive low-energy $e^{+} e^{-} \rightarrow$ hadrons final states. These measurements are needed as input in order to determine the standard model prediction for the muon anomalous magnetic moment $g-2$, a topic of great current interest because of the observed discrepancy of around 3.5 standard deviations between this prediction and the data [4]. The study of these cross sections is also interesting in its own right, providing precise new information on low-energy hadron spectroscopy and decay rates.

The study is based on the initial-state radiation (ISR) method to measure low-energy cross sections. A photon is radiated from either the incoming electron or positron in an $e^{+} e^{-}$ annihilation. This lowers the effective c.m. energy of the hadronic system formed in the annihilation. The rate of final-state radiation is negligible. In the BABAR studies, the ISR photon is identified as the highest energy photon in an event, and is required to have a c.m. energy larger than $3 \mathrm{GeV}$. The event signature is thus a high energy photon, with hadrons recoiling against the photon. This gives an easily recognizable event signature with high acceptance and low background. Because the energy and momentum carried away by the ISR photon differs from event to event, one can access a wide range of effective c.m. energies for the hadronic system: from threshold up to around $5 \mathrm{GeV}$. 
The study of intermediate states within the hadronic system is also interesting and important. It sheds light on the hadron production process and can be used to test theoretical predictions. From a practical point of view, it is also important: knowledge of the intermediate resonance structure can be incorporated into the MC simulations to reduce systematic uncertainties in the experimental acceptance and reconstruction efficiencies, which depend on the intermediate states since different intermediate resonances lead to different distributions of energy and angles in the final-state particles, and thus to different acceptances and efficiencies.

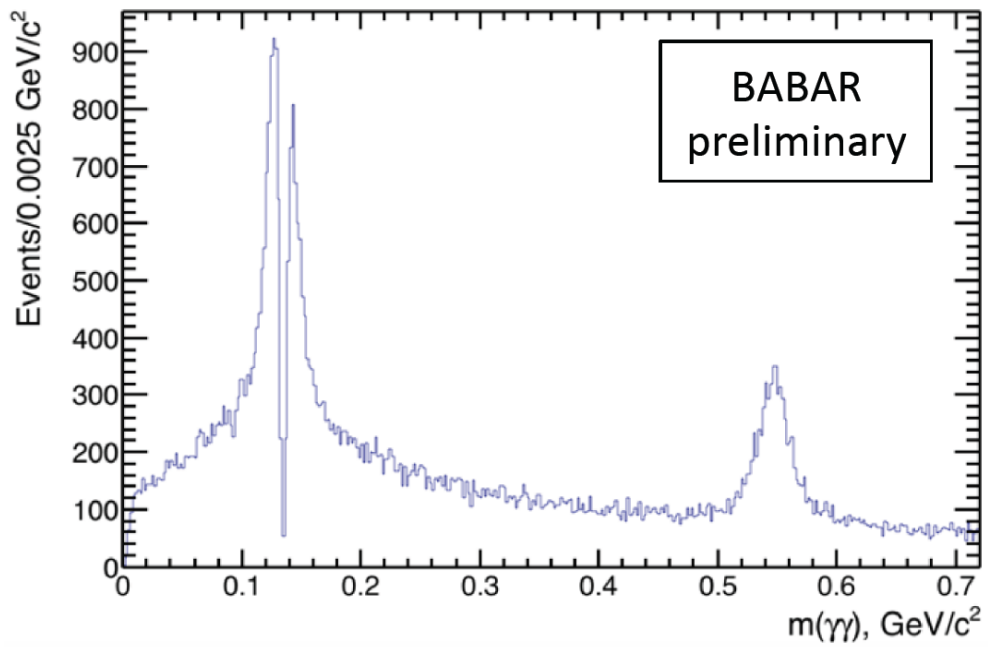

Figure 7. Invariant mass of the third photon pair in the selected $e^{+} e^{-} \rightarrow \pi^{+} \pi^{-} \pi^{0} \pi^{0} \gamma \gamma$ events.

The selection of the $e^{+} e^{-} \rightarrow \pi^{+} \pi^{-} \pi^{0} \pi^{0} \pi^{0}$ and $\pi^{+} \pi^{-} \pi^{0} \pi^{0} \eta$ events begins by requiring that an event contain exactly two charged tracks of opposite sign that are inconsistent with being a kaon or muon and that extrapolate to near the interaction point. An ISR photon candidate and at least six other photon candidates must be present. The six non-ISR photon candidates are combined into three pairs (15 different possible combinations for each set of six examined photons). The diphoton invariant mass of two of the pairs must be consistent with the $\pi^{0}$ mass. A kinematic fit is performed to the $e^{+} e^{-} \rightarrow \pi^{+} \pi^{-} \pi^{0} \pi^{0} \gamma \gamma$ signal hypothesis, constraining two $2 \gamma$ combinations to the $\pi^{0}$ mass and treating the remaining two photons as being independent. That way, both the $\pi^{+} \pi^{-} \pi^{0} \pi^{0} \pi^{0}$ and $\pi^{+} \pi^{-} \pi^{0} \pi^{0} \eta$ events are selected. The combination of the six photons that yields the smallest $\chi_{2 \pi 2 \pi^{0} 2 \gamma \gamma_{I S R}}^{2}$ is selected, with a requirement that $\chi_{2 \pi 2 \pi^{0} 2 \gamma \gamma_{I S R}}^{2}<60$. Background is rejected by performing the analogous fit to the chief background process, $e^{+} e^{-} \rightarrow \pi^{+} \pi^{-} \pi^{0} \pi^{0} \gamma_{I S R}$, and requiring the corresponding $\chi^{2}$ to exceed 30. The invariant mass of the third photon pair in the selected events in shown in Fig. 7. Peaks at the $\pi^{0}$ and $\eta$ masses are clearly visible. The double peak at the $\pi^{0}$ mass is an artifact of the $\pi^{0}$ mass constraint.

The background is subtracted using 1) a data sideband defined by $60<\chi_{2 \pi 2 \pi^{0} 2 \gamma \gamma_{I S R}}^{2}<$ 120 to evaluate ISR backgrounds and 2) simulation normalized to data using data control regions to evaluate non-ISR backgrounds. The cross section for $e^{+} e^{-} \rightarrow \pi^{+} \pi^{-} \pi^{0} \pi^{0} \pi^{0}$ is then calculated as

$$
\sigma_{2 \pi 3 \pi^{0}}\left(E_{\text {c.m. }}\right)=\frac{d N_{2 \pi 3 \pi^{0}}\left(E_{\text {c.m. }}\right)}{d \mathcal{L}\left(E_{\text {c.m. }}\right) \epsilon\left(E_{\text {c.m. }}\right)\left(1+\delta_{R}\right)}
$$



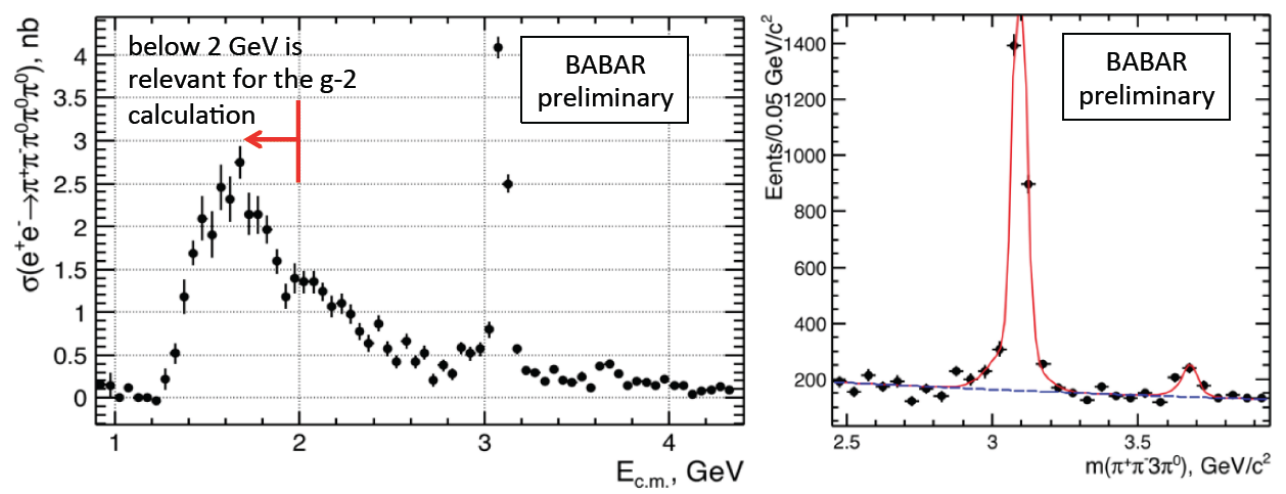

Figure 8. The measured $e^{+} e^{-} \rightarrow \pi^{+} \pi^{-} \pi^{0} \pi^{0} \pi^{0}$ cross section. The right-hand plot shows an expanded view around the charmonium region.
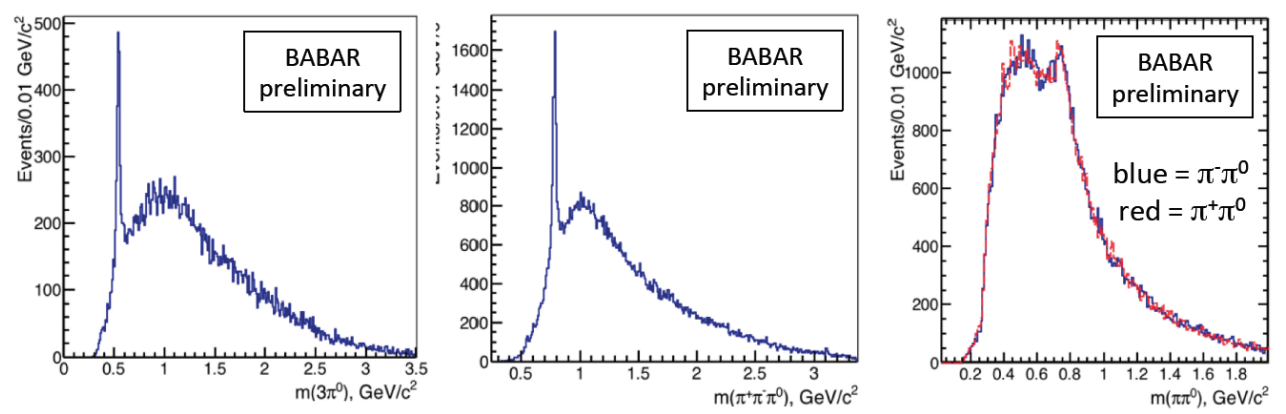

Figure 9. The dominant intermediate resonance structure observed in $e^{+} e^{-} \rightarrow \pi^{+} \pi^{-} \pi^{0} \pi^{0} \pi^{0}$ events: peaks at the (left) $\eta \rightarrow 3 \pi^{0}$, (center) $\omega \rightarrow \pi^{+} \pi^{-} \pi^{0}$, and (right) $\rho^{ \pm} \rightarrow \pi^{ \pm} \pi^{0}$ masses.

where $d \mathcal{L}$ is the effective integrated luminosity at hadronic system c.m. energy $E_{\text {c.m. }}, \epsilon$ is the reconstruction efficiency, and the radiative correction $1+\delta_{R}$ accounts for final-state photon radiation.

The resulting $e^{+} e^{-} \rightarrow \pi^{+} \pi^{-} \pi^{0} \pi^{0} \pi^{0}$ cross section in shown in Fig. 8. The cross section below around $2 \mathrm{GeV}$ is the data that is relevant for the muon $g-2$ calculation. This is the first measurement of this cross section. Clear $J / \psi$ and $\psi(2 S)$ peaks are visible. The corresponding branching fractions, measured for the first time, are $\mathcal{B}\left(J / \psi \rightarrow \pi^{+} \pi^{-} 3 \pi^{0}\right)=(27.0 \pm 2.8) \times 10^{-3}$ and $\mathcal{B}\left(\psi(2 \mathrm{~S}) \rightarrow \pi^{+} \pi^{-} 3 \pi^{0}\right)=(5.2 \pm 0.9) \times 10^{-3}$. The intermediate state structure is studied and is found to be dominated by 1) $e^{+} e^{-} \rightarrow \eta \pi^{+} \pi^{-}$(including $e^{+} e^{-} \rightarrow \eta \rho$ ), with $\eta \rightarrow \pi^{0} \pi^{0} \pi^{0}$ (Fig. 9 left), 2) $e^{+} e^{-} \rightarrow \omega \pi^{0} \pi^{0}$ with $\omega \rightarrow \pi^{+} \pi^{-} \pi^{0}$ (Fig. 9 center), and 3) $e^{+} e^{-} \rightarrow \rho^{ \pm} \pi^{\mp} \pi^{0} \pi^{0}$ with $\rho^{ \pm} \rightarrow \pi^{ \pm} \pi^{0}$ (Fig. 9 right).

The analogous procedure is followed to measure the $e^{+} e^{-} \rightarrow \pi^{+} \pi^{-} \pi^{0} \pi^{0} \eta$ cross section. The result is shown in Fig. 10. A clear $J / \psi$ peak is seen. The result for the $J / \psi$ branching fraction is $\mathcal{B}\left(J / \psi \rightarrow \pi^{+} \pi^{-} \pi^{0} \pi^{0} \eta\right)=(2.3 \pm 0.5) \times 10^{-3}$. We do not observe a significant $\psi(2 S)$ signal in this channel and set a limit at $90 \%$ confidence level $\mathcal{B}\left(\psi(2 \mathrm{~S}) \rightarrow \pi^{+} \pi^{-} \pi^{0} \pi^{0} \eta\right)<$ $0.35 \times 10^{-3}$. These are the first measurements of this cross section and of the corresponding $J / \psi$ branching fraction. The intermediate resonance structure is dominated by 1) $e^{+} e^{-} \rightarrow$ 


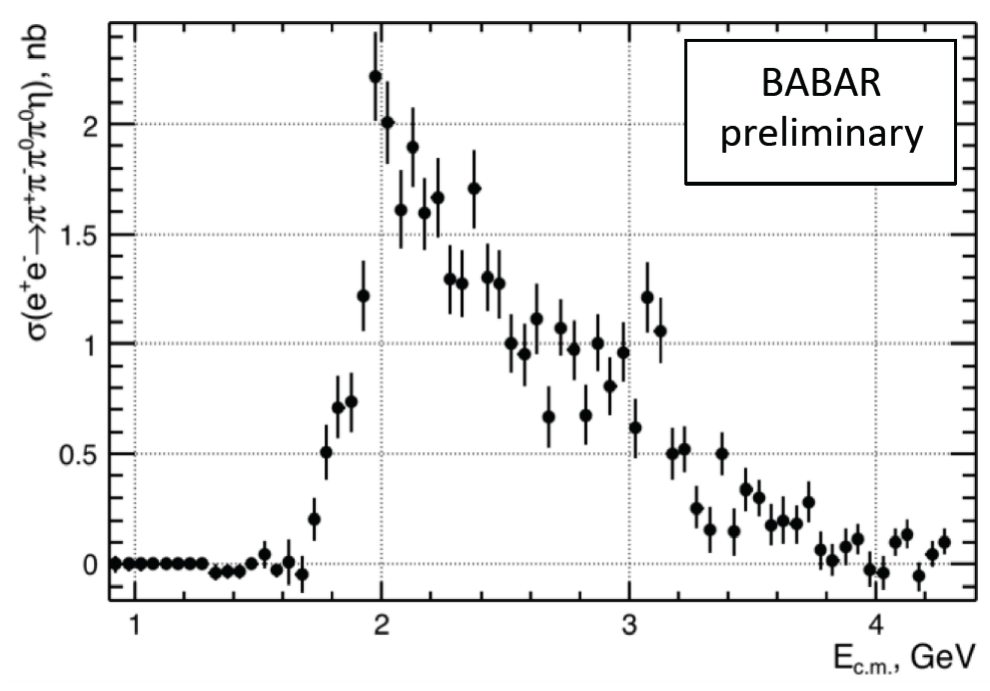

Figure 10. The measured $e^{+} e^{-} \rightarrow \pi^{+} \pi^{-} \pi^{0} \pi^{0} \eta$ cross section.
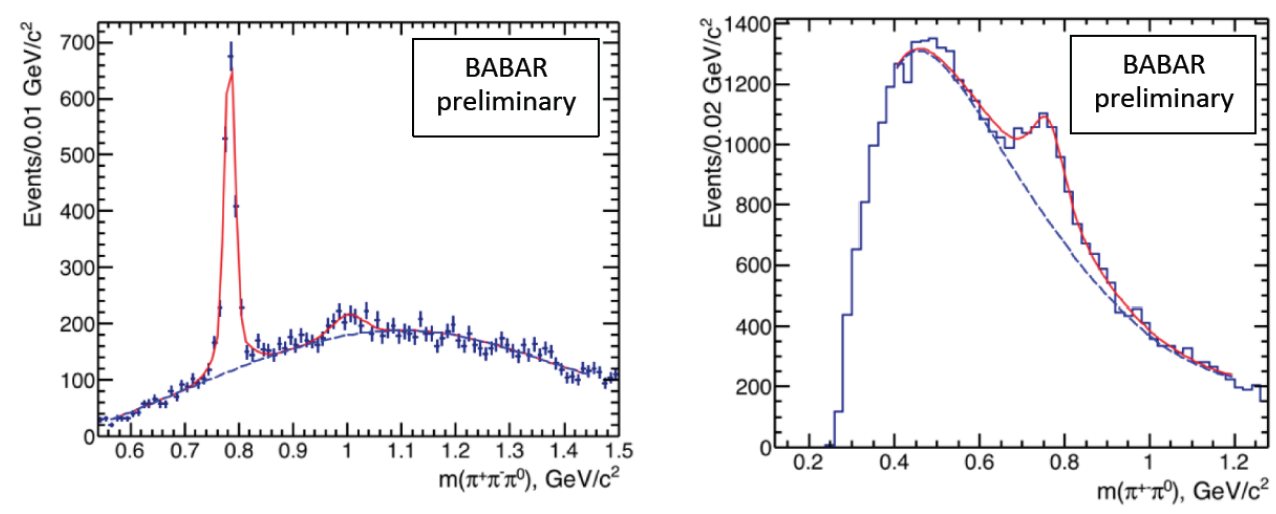

Figure 11. The dominant intermediate resonance structure observed in $e^{+} e^{-} \rightarrow \pi^{+} \pi^{-} \pi^{0} \pi^{0} \eta$ events: peaks at the (left) $\omega$ and $\phi \rightarrow \pi^{+} \pi^{-} \pi^{0}$ and (right) $\rho^{ \pm} \rightarrow \pi^{ \pm} \pi^{0}$ masses.

$\omega \pi^{0} \eta$ and $e^{+} e^{-} \rightarrow \phi \pi^{0} \eta$ with $\omega, \phi \rightarrow \pi^{+} \pi^{-} \pi^{0}$ (Fig. 11 left) and 2) $e^{+} e^{-} \rightarrow \rho^{ \pm} \pi^{\mp} \pi^{0} \eta$ with $\rho^{ \pm} \rightarrow \pi^{ \pm} \pi^{0}$ (Fig. 11 right).

\section{Summary}

Recent BABAR results on low-energy exclusive hadronic final states produced in $e^{+} e^{-}$collisions have been presented. A first measurement of the $e^{+} e^{-} \rightarrow e^{+} e^{-} \eta^{\prime}$ process in the doubletag mode has been made and the corresponding $\gamma^{*} \gamma^{*} \rightarrow \eta^{\prime}$ transition form factor (TFF) determined. The TFF measurements agree with the predictions of perturbative QCD but not with the predictions of the vector dominance model. This result represents a novel test of QCD. Measurements of low-energy exclusive hadronic cross sections are needed for the muon $g-2$ standard model calculation and yield important information on hadron dynamics. 
They allow tests of QCD, including for the total $e^{+} e^{-}$hadronic cross section at a center-ofmass energy around $2 \mathrm{GeV}$. The first measurements of or results on the $e^{+} e^{-} \rightarrow \pi^{+} \pi^{-} \pi^{0} \pi^{0} \pi^{0}$ and $\pi^{+} \pi^{-} \pi^{0} \pi^{0} \eta$ cross sections, the corresponding $J / \psi$ and $\psi(2 \mathrm{~S})$ branching fractions, and the intermediate resonance structure have been presented.

\section{References}

[1] BABAR Collaboration, J. P. Lees et al., BABAR-PUB-18/006, arXiv:1808.08038, submitted to Phys. Rev. D.

[2] CLEO Collaboration, J. Gronberg et al. Phys. Rev. D 57 (1998) 33.

[3] BABAR Collaboration, P. del Amo Sanchez et al., Phys. Rev. D 84 (2011) 052001.

[4] M. Davier, A. Hoecker, B, Malaescu, and Z. Zhang, Z. Phys. C 71, 1515 (2011). 